\title{
Antimicrobial Resistance, Virulence Factors, Diversity, and Genetic Clonality of Enterococcus Species from Clinical Samples
}

\section{Arezoo Asadi $^{1,2}$, Enayati Mohsen ${ }^{1}$, Atieh Darbandi, ${ }^{1,2}$, Hamid Pajavand ${ }^{3}$ and Malihe Talebi ${ }^{1,2 *}$}

${ }^{1}$ Department of Microbiology, School of Medicine, Iran University of Medical

Sciences, Tehran, Iran

${ }^{2}$ Microbial Biotechnology Research Centre, Iran University of Medical Sciences,

Tehran, Iran

${ }^{3}$ Department of Microbiology, School of Medicine, Tarbiat Modares University of

Medical Sciences, Tehran, Iran

*Corresponding Author: Malihe Talebi, Department of Microbiology, School of Medicine and Microbial Biotechnology Research Centre, Iran University of Medical Sciences, Shahid Hemmat Highway, Tehran, Iran.. E-mail: talebi.m@iums.ac.ir, talebi_25@yahoo.com
Received: February 12, 2021

Published: June 24, 2021

(C) All rights are reserved by Arezoo Asadi., et al.

\begin{abstract}
Over the past decade, the emergence of antibiotic-resistant enterococci has posed critical challenges to the treatment of nosocomial infections around the world, and VRE infections have been associated with mortality and morbidity worldwide, especially in immunocompromised individuals. A total of 285 Enterococcus isolates were collected from patients referring to three major hospitals in Tehran from July to December 2012, and species were identified using biochemical tests and PCR. PCR assay and antibiotic susceptibility testing were performed to detect Enterococcus species, virulence factors, and resistance genes as well as to evaluate drug resistant isolates, respectively. The clonality of the isolates was also determined by PFGE. The isolates were found to consist of $E$. faecalis (65\%) and E. faecium (31\%) species. According to the results, 39 and $53 \%$ of E. faecalis and E. faecium isolates were resistant to the most commonly used antimicrobial agents, respectively. Also, acm $(23,64 \%)$ and hyl $(6,86 \%)$ were the most prevalent genes in E. faecalis isolates, while the most prevalent virulence genes in E. faecium isolates were cyt (7, 100\%), gel (30, 97\%), and asa (29, 97\%). PFGE revealed a high heterogeneity among 50 VRE isolates. The emergence of multidrug-resistant and heterogeneous populations of enterococci is considered as a serious threat of global concern.
\end{abstract}

Keywords: Enterococcus; Resistance; Antimicrobials; PFGE

\section{Abbreviations}

BSI: Bloodstream Infections; NNIS: National Nosocomial Infection Surveillance; GRE: Glycopeptide-resistant Enterococci; VRE: Vancomycin-resistant Enterococci; ICU: Intensive Care Unit; PYR: Pyrrolidonyl Arylamidase; MDR: Multidrug Resistant; BHI: Brain Heart Infusion; PFGE: Pulsed-field Gel Electrophoresis

\section{Introduction}

The increasing emergence of enterococci as one of the chief causes of nosocomial infections and their high capacity to receive and transfer antibiotic resistance genes have brought members of this genus, especially Enterococcus faecium, to the forehand of hospital infection control issues. Enterococci are considered as the second leading cause of urinary tract infections [1] as well as one of the causes of nosocomial UTI (10\%), intra-abdominal and pelvic abscesses, and post-surgery wound infections [2,3]. Studies have shown that Enterococcus is the fourth and fifth most common cause of bloodstream infections (BSI) in North America (10.2\%) and Europe $(7.2 \%)$, respectively, as assayed by the SENTRY Antimicrobial Surveillance scheme [4]. According to the National Nosocomial Infection Surveillance (NNIS), the incidence of glycopeptide-resistant enterococci (GRE) increased from $0.3 \%$ in 1989 to $25.9 \%$ in 1999 in the USA, which could be considered as a serious threat [5]. 
In recent years, the emergence of vancomycin-resistant enterococci (VRE) has posed critical challenges for practitioners responsible for controlling hospital infections as well as for clinicians remedying patients with enterococcal nosocomial infections [6], and VRE infections have been associated with mortality and morbidity worldwide, especially in immunocompromised individuals; also, nearly $25 \%$ of enterococcal infections in intensive care unit (ICU) patients have been detected to be VRE infections [7]. Increased consumption of vancomycin for the treatment of methicillin-resistant staphylococcal infections has been identified as a prominent factor in the development of VRE, among other factors such as clonal dissemination [8]. Enterococcal infections in hospitals are mostly clonal with epidemic-virulent clonal complex 17 (CC-17) lineage of E. faecium [9]. Some of which are caused by single strain outbreak, while others are caused by simultaneous clonal spread of endemic clusters existing in hospitals [8]. Control and prevention programs designed for hospital-acquired infections caused by enterococci concentrate on procedures that hinder cross-transmission between cases and control antimicrobials consummation [10].

According to diverse reports on VRE prevalence rate in health centers in Iran, diseases and mortality rate due to enterococcal infections in Iran are increasing every day. One of the most important reasons is that the proper antimicrobial treatment of enterococcal infections has gradually become more difficult for Iranian physicians due to the lack of sufficient information about the prevalence of VRE and increased antibiotic resistance in these strains [11]. Therefore, this survey aimed to investigate the frequency of Enterococcus species, pathogenic factors, and VRE isolates as well as VRE antibiotic resistance pattern in three different healthcare departments in Tehran.

\section{Material and Methods}

Samples collection and identification

In this cross-sectional study, 285 Enterococcus isolates were collected from patients referring to three major hospitals in Tehran from July to December 2012. Samples (taken from urine, catheter, BAL, blood and wound) were instantly cultured on $\mathrm{m}$-Enterococcus agar, bile esculin agar, and blood agar media at $37^{\circ} \mathrm{C}$. Enterococcus genus identification was performed based on the following microbiological tests: Gram stain, catalase, presence of pyrrolidonyl arylamidase (PYR), and growth on bile-aesculin agar medium with $6.5 \% \mathrm{NaCl}$. Other tests performed in this study were as follows: motility, arginine decarboxylation in Moeller decarboxylase media, pyruvate utilization, and carbohydrates fermentation tests (Arabinose, Raffinose, Mannitol, Ribose).

\section{Antibiotic susceptibility testing}

All Enterococcus isolates were tested for susceptibility to Vancomycin $30(\mu \mathrm{g})$, Gentamicin $120(\mu \mathrm{g})$, Erythromycin $15(\mu \mathrm{g})$, Amoxicillin $25(\mu \mathrm{g})$, Ciprofloxacin $5(\mu \mathrm{g})$, Streptomycin $30(\mu \mathrm{g})$, Linozolid $30(\mu \mathrm{g})$, Synercid $15(\mu \mathrm{g})$, Ampicillin $10(\mu \mathrm{g})$, Chloramphenicol 30 $(\mu \mathrm{g})$, Nitrofurantoin $300(\mu \mathrm{g})$ and Rifampin $5(\mu \mathrm{g})$ by the KirbyBauer disk diffusion method (MAST, United Kingdom) [12]. Resistance breakpoints to a particular antibiotic were determined using standard reference values. Isolates that were resistant to at least one antibiotic in three or more antibiotic classes were classified as multidrug resistant (MDR) [13]. Staphylococcus aureus ATCC 25923 was included as a quality control organism for antimicrobial susceptibility determination.

\section{DNA extraction}

Enterococcus strains were grown overnight at $35^{\circ} \mathrm{C}$ on Brain Heart Infusion (BHI) agar. Then two or three colonies were scraped from the surface of each agar culture plate, and the pellet was then used for DNA extraction by High Pure PCR Template Preparation Kit (Roche Diagnostic GmbH, Mannheim, Germany) [14].

PCR assay for the detection of E. faecium, E. faecalis, and virulence factors

According to table 1, all of the 285 enterococci isolates were analyzed for the presence of specific genes by PCR. Singleplex PCR was carried out for the amplification of the D-Ala-D-Ala ligase $(d d l)$ gene to detect E. faecium and E. faecalis, Singleplex PCR was also carried out for the amplification of vanA and vanB1 as resistance genes, virulence factors were detected using multiplex PCR for acm, gelE, Hyl, asa, esp, and cyt genes. PCR reactions were performed in a $25 \mu \mathrm{L}$ volume consisting of $2.5 \mu \mathrm{L}$ of PCR Buffer (10X), $0.6 \mu \mathrm{L}$ of $\mathrm{MgCl} 2(50 \mathrm{mM}), 0.4 \mu \mathrm{L}$ of dNTP Mix $(10 \mathrm{mM}), 0.4 \mu \mathrm{L}$ of primer $1(100 \mathrm{pm} / \mu \mathrm{L}), 0.1 \mu \mathrm{L}$ of Taq DNA polymerase $(5 \mathrm{U} / \mu \mathrm{L}), 1 \mu \mathrm{l}$ of Template DNA, and $19.6 \mu \mathrm{L}$ of $\mathrm{ddH}_{2} \mathrm{O}$.

PCR cycling conditions were as follows: an initial denaturation step of DNA at $95^{\circ} \mathrm{C}$ for $4 \mathrm{~min}$, followed by 30 cycles of denaturation at $95^{\circ} \mathrm{C}$ for $30 \mathrm{~s}$, primer annealing at 50 to $63^{\circ} \mathrm{C}$ (according to table 1) for $1 \mathrm{~min}$, DNA synthesis step at $72^{\circ} \mathrm{C}$ for $1 \mathrm{~min}$, and final DNA 


\begin{tabular}{|c|c|c|c|c|}
\hline $\begin{array}{l}\text { Virulence } \\
\text { Genes }\end{array}$ & Primer Sequence $\left(5^{\prime}-3^{\prime}\right)$ & $\begin{array}{c}\text { Size Products } \\
\text { (bp) }\end{array}$ & $\begin{array}{c}\text { Annealing } \\
\text { Temperature }\end{array}$ & Reference \\
\hline ddl(E. faecalis) & $\begin{array}{l}\text { F-TTGAGGCAGACCAGATTGACG } \\
\text { R- TATGACAGCGACTCCGATTCC }\end{array}$ & 658 & $50 \stackrel{\circ}{ } \mathrm{C}$ & {$[30]$} \\
\hline ddl(E. faecalis) & $\begin{array}{l}\text { F- ATCAAGTACAGTTAGTCT } \\
\text { R- ACGATTCAAAGCTAACTG }\end{array}$ & 941 & $50 \stackrel{\circ}{ } \mathrm{C}$ & {$[31]$} \\
\hline acm & $\begin{array}{l}\text { F- GGCCAGAAACGTAACCGATA } \\
\text { R- AACCAGAAGCTGGCTTTGTC }\end{array}$ & 135 & $52 \stackrel{\circ}{\circ}$ & [32] \\
\hline gelE & $\begin{array}{l}\text { F- TATGACAATGCTTTTTGGGAT } \\
\text { R- AGATGCACCCGAAATAATATA }\end{array}$ & 213 & $52 \stackrel{\circ}{\circ}$ & {$[32]$} \\
\hline Hyl & $\begin{array}{l}\text { F- ACAGAAGAGCTGCAGGAAATG } \\
\text { R- GACTGACGTCCAAGTTTCCAA }\end{array}$ & 276 & $52 \stackrel{\circ}{\circ}$ & [33] \\
\hline asa & $\begin{array}{l}\text { F- GCACGCTATTACGAACTATGA } \\
\text { R- TAAGAAAGAACATCACCACGA }\end{array}$ & 375 & $52 \stackrel{\circ}{\circ}$ & [33] \\
\hline Esp & $\begin{array}{l}\text { F- AGATTTCATCTTTGATTCTTGG } \\
\text { R- AATTGATTCTTTAGCATCTGG }\end{array}$ & 510 & $52 \stackrel{\circ}{ } \mathrm{C}$ & [34] \\
\hline Cyt & $\begin{array}{l}\text { F- AGATTTCATCTTTGATTCTTGG } \\
\text { R- AATTGATTCTTTAGCATCTGG }\end{array}$ & 688 & $52 \stackrel{\circ}{ } \mathrm{C}$ & {$[34]$} \\
\hline $\operatorname{Van} A$ & $\begin{array}{l}\text { FCATGAATAGAATAAAAGTTGCAATA } \\
\text { R- CСССТTTAACGCTAATACGATCAA }\end{array}$ & 1030 & $54 \stackrel{\circ}{\circ} \mathrm{C}$ & {$[34]$} \\
\hline $\operatorname{Van} B$ & $\begin{array}{l}\text { FGTGACAAACCGGAGGCGAGGA } \\
\text { R-CCGCCATCCTCCTGCAAAAAA }\end{array}$ & 433 & $54 \stackrel{\circ}{\circ} \mathrm{C}$ & {$[34]$} \\
\hline
\end{tabular}

Table 1: Primers used to detect E. faecium and E. faecalis, resistance genes and virulence factors.

synthesis at $72^{\circ} \mathrm{C}$ for $10 \mathrm{~min}$ [15]. Eventually, $10 \mu \mathrm{L}$ of PCR products were analyzed by electrophoresis (120 mV for $80 \mathrm{~min}$ ) on a $1 \%$ agarose gel using safe stain. The agarose gel was illuminated by UV, and DNA bands were finally analyzed and recorded.

Pulsed-field gel electrophoresis (PFGE)

Enterococcus isolates were analyzed by pulsed-field gel electrophoresis (PFGE). First all selected Enterococcus strains grew in $\mathrm{BHI}$ broth overnight with shaking at $37^{\circ} \mathrm{C}$. All the cultures were centrifugate and cells were re-suspended in $5 \mathrm{ml}$ of TE buffer (10 mM Tris, 0.1 mM EDTA [pH 8.0]), blended with an equalized volume of $2 \%$ low melting agarose (Bio-Rad, Hercules, CA) in distilled water and poured down into a plug mold. Then, all the plugs were treated in lysis buffer including lysosome $(1 \mathrm{mg} / \mathrm{ml})$ and RNAS (5 $\mathrm{mh} / \mathrm{ml}$ ), (6 mM Tris [pH 7.5], $100 \mathrm{mM}$ EDTA [pH7.5], $1 \mathrm{M} \mathrm{Na-Cl,}$ $0.5 \%$ Brij-58, $0.2 \%$ sodium deoxycholate, and $1 \%$ sodium lauryl sarcosine) overnight at $37^{\circ} \mathrm{C}$. Mixtures were supplanted by ES solution (0.5 M EDTA [pH 9.5], 1\% sarcosine) and then ESP solution 
(0.5 M EDTA [pH 9.5], 1\% sarcosin, and $0.5 \mathrm{mg}$ of proteinase $\mathrm{K} /$ $\mathrm{ml}$ ) and were incubated $48 \mathrm{~h}$ at $50^{\circ} \mathrm{C}$. Then the plugs were washed with TE buffer and stored at $4^{\circ} \mathrm{C}$. Afterwards, the plugs were digested with $20 \mathrm{U}$ Smal (Roche, Manhiem, Germany) and settled in the wells of $1 \%$ agarose in $0.5 \% \times$ TBE and electrophoreses with switch times ramped from 5 to $35 \mathrm{~s}$ at $6 \mathrm{~V}$ with a run time of $27 \mathrm{~h}$ at $14^{\circ} \mathrm{C}$ in the Bio-Rad CHEF-DRIII system. DNA from Salmonella choleraeraesuis serotype Branderup H9812 (Pulsenet, www.cdc. gov/pulsenet) were used as molecular size marker. The gels were stained with ethidium bromide and visualized the restricted DNA with ultraviolet light PFGE patterns then analyzed and interpreted using GelCompar II software Version 6.5 (Applied Maths, Belgium). The genetic similarities between the fingerprints were determined using Dice coefficients and unweighted pair-group method with arithmetic mean (UPGMA) [16].

\section{Statistical analyses}

Data were statistically analyzed by employing Pearson chisquare test for dependent samples or Fisher's exact test where appropriate using SPSS software Version 19.0. A $p$-value of $<.05$ was considered as statistically significant.

\section{Results and Discussion}

Out of 285 Enterococcus isolates obtained from 5560 hospitalized patients with different age groups (from infants to 93 years), 168 (65\%) were from Milad hospital, 67 (24\%) were from Shariati hospital, and 32 (11\%) were from Tehran Heart Center. The majority of the isolate were recovered from urine $(196,69 \%)$ and blood $(35,12 \%)$ samples of both males (48\%) and females (52\%) (Table 2).

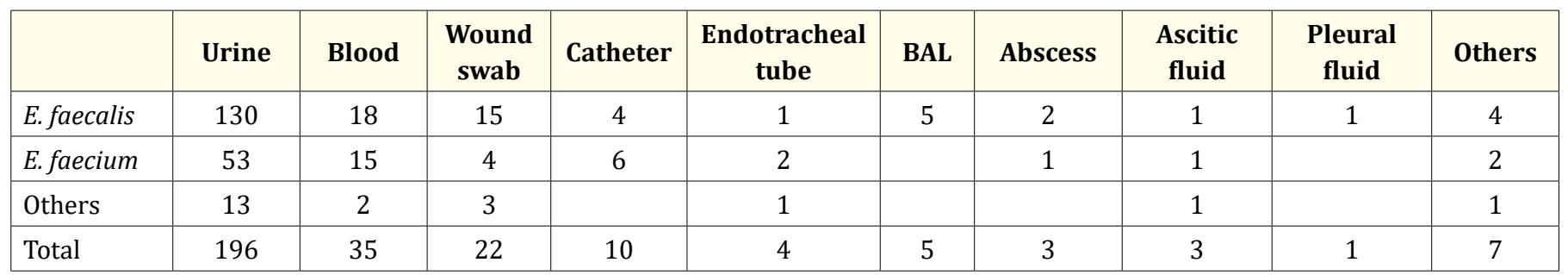

Table 2: Distribution of Enterococcus strains in different clinical samples.

In this study, two different Enterococcus species were identified based on the standard guideline of species identification. Most of the isolates were E. faecalis (65\%), followed by E. faecium (31\%) and other strains (4\%). The distribution of Enterococcus strains in different clinical wards are shown in table 3.

\section{Antibiotic susceptibility testing}

Resistance of $E$. faecium isolates to antimicrobial agents tested in this study based on DDT (disk diffusion technique) was higher than that of $E$. faecalis isolates, except for ampicillin and amoxicillin. The highest susceptibility of E. faecium isolates was observed to ampicillin (79\%) and amoxicillin (67\%), while the highest susceptibility of E. faecalis isolates was observed to nitrofurantoin (86\%) and synercid (86\%). Antimicrobial resistance patterns of Enterococcus isolates are shown in table 4.

Out of 285 Enterococcus isolates, 50 (17.5\%) were found to be VRE using DDT. Overall, the prevalence of vancomycin-resistant $E$. faecium $(37,74 \%)$ isolates were higher than that of vancomycinresistant E. faecalis $(13,26 \%)$ isolates. In this study, 38 (13.3\%) isolates were multidrug resistant (MDR). The prevalence of MDR isolates was higher among E. faecium $(20,53 \%)$ isolates compared to E. faecalis $(15,39 \%)$ isolates.

Prevalence of virulence genes among Enterococcus strains

Figure 1 shows the prevalence of six virulence genes identified in the sample. According to the results, acm $(23,64 \%)$ and hyl (6, $86 \%$ ) were the most prevalent genes in E. faecalis isolates, while the most prevalent virulence genes in E. faecium isolates were cyt (7, 100\%), gel $(30,97 \%)$, and asa $(29,97 \%)$. Only two (6\%) E. faecalis isolates carried asa and gel genes, and cyt was not detected in E. faecalis isolates.

\section{Pulsed-field gel electrophoresis (PFGE)}

The clonality of 50 VRE E. faecium and E. faecalis isolates were analyzed by PFGE. Then found among all VRE isolates 8 types con- 


\begin{tabular}{|l|c|c|c|c|}
\hline & E. faecalis & E. faecium & Others & Total \\
\hline CCU & 16 & 4 & 4 & 24 \\
\hline ICU & 24 & 26 & 2 & 52 \\
\hline NICU & 1 & 0 & 0 & 1 \\
\hline Lurgery & 9 & 6 & 2 & 17 \\
\hline Children & 2 & 0 & 0 & 2 \\
\hline Hospitalization & 14 & 5 & 1 & 13 \\
\hline Bone marrow & 4 & 3 & 0 & 19 \\
\hline Transplant & 14 & 5 & 1 & 20 \\
\hline Internal & 47 & 16 & 5 & 68 \\
\hline Outpatient & 2 & 0 & 0 & 2 \\
\hline Glands & 3 & 1 & 0 & 4 \\
\hline Heart & 19 & 4 & 3 & 26 \\
\hline Kidney & 4 & 4 & 0 & 8 \\
\hline Digestion & 0 & 2 & 0 & 2 \\
\hline Neurology & 18 & 0 & 2 & 20 \\
\hline Emergency & 180 & 85 & 20 & 285 \\
\hline Total & & & & \\
\hline
\end{tabular}

Table 3: Distribution of Enterococcus strains in different clinical wards.

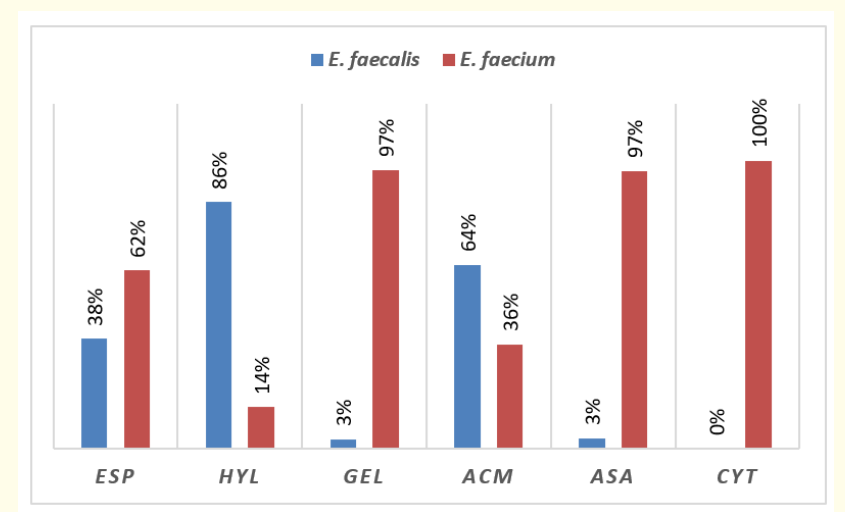

Figure 1: Prevalence and distribution of virulence factors in Enterococcus isolates.

taining 50 identical isolates with more than $80 \%$ similarity (Figure 2). The remaining two types contained highly diverse isolates belonging to 2 single types (ST, ST2). Figure 2 shows the representative PFGE patterns. Three dominant clusters (CT, CT6, CT8) containing 7, 8 and 8 isolates respectively. In CT1 and CT2 types, there were some isolates from all three hospitals, suggesting the rotation of this bacterium in these three hospitals. The isolates typing results in this study showed that there was the possibility of in-hospital transfer of strains.

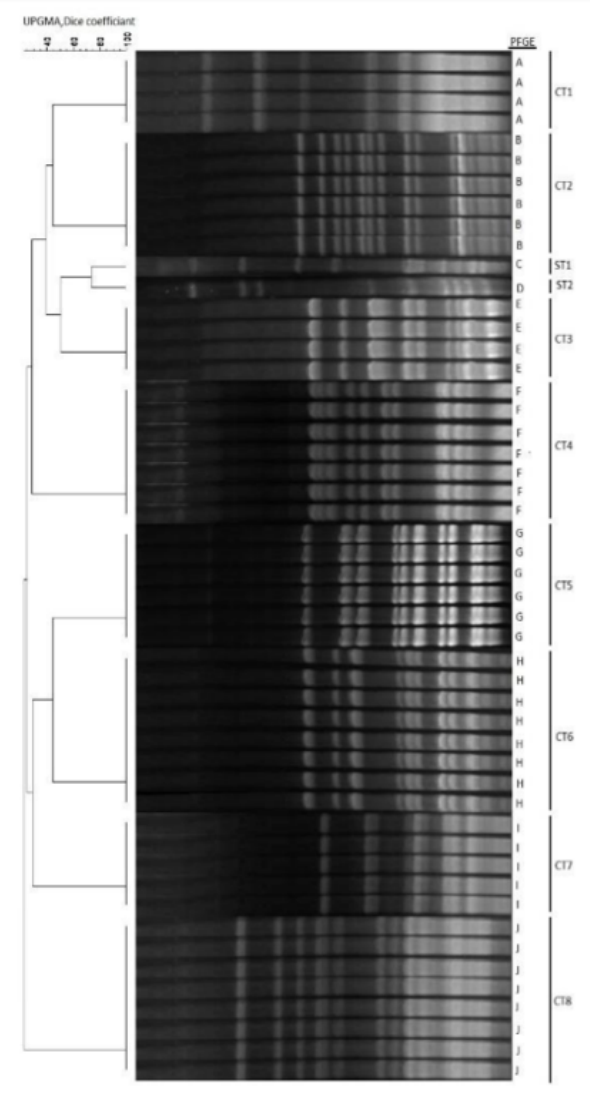

Figure 2: Dendrogram based on pulsed-field gel electrophoresis (PFGE) SmaII restriction pattern analysis of VRE isolates.

Enterococcus are part of the normal flora of the oral cavity, intestinal tract, vagina, etc. They are associated with different types of infections, especially as nosocomial pathogens. The emergence of enterococcal resistance and tolerance against antimicrobial agents is a serious concern worldwide [17]. Therefore, it is important to know enterococcus resistance patterns and antibiogram profile. In 
this study, 285 Enterococcus isolates were recovered from 5560 clinical specimens during a six-month study period. The samples examined were urine, blood, catheter, BAL, and wound samples taken from hospitalized patients. The highest number of isolates was obtained from urine (69\%), followed by blood (12\%) samples. Enterococcal bacteraemia mortality rates have been reported from 19 to $48 \%$. It is generally accepted that enterococcal bacteraemia often occurs in patients with blood stream and surgical site infections. Enterococcus are next to Staphylococcus aureus and coagulase-negative Staphylococcus causing clinically significant infections in the bloodstream and other tissues; therefore, their isolation from bloodstream is very important and is often very difficult for a laboratory expert [18]. Furthermore, out of 285 isolates, $52 \%$ were non hemolytic, and $48 \%$ were $\beta$ hemolytic. Hemolytic activity plays an important role in the pathogenesis of enterococci. The majority of the $\beta$ hemolytic isolates were obtained from urine specimens. Most of the isolates investigated were E. faecalis (65\%), followed by E. faecium (31\%) and other strains (4\%). Like other studies in Iran, E. faecalis was more prevalent among the isolates investigated in this study [19]. In a study in India, out of 80 Enterococcus strains, 69 were E. faecalis, 10 were E. faecium, and one was E. durans [20]. When enterococci are isolated in different geographical regions, there might be variation in species distribution. E. faecalis and E. faecium are the most frequent enterococcal species isolated from different clinical specimens, they are responsible for $90 \%$ of nosocomial infections [21]. The prevalence of resistance to chloramphenicol, nitrofurantoin, and synercid in E. faecalis isolates as well as to ampicillin and amoxicillin in E. faecium isolates was low, indicating that these antimicrobial agents could be considered as therapeutic options. Resistance rate to ampicillin among E. faecalis isolates was higher than in E. faecium isolates (74\% versus $21 \%$ ); this finding is different from the finding of another study by Saifi., et al. [19]. Enterococcus could overexpress low-affinity penicillinbinding proteins against $ß$-lactams. In addition, plasmid mediated ß-lactamases have been shown to be responsible for resistance in these strains, allowing Enterococcus to be intrinsically resistant to penicillin [22]. Dalfopristin resistance in E. faecalis strains is conferred through an efflux pump that seems to be intrinsic in this species. MDR strains in the present study were reported to be $13.3 \%$. The prevalence of MDR isolates was higher in E. faecium $(20,53 \%)$ than in E. faecalis $(15,39 \%)$ isolates. Numerous factors such as antimicrobials overuse, genetic mutations, and disease control measures contribute to the development of multidrug resistance phenotypes [23]. MDR enterococcus exhibiting high resistance to penicillin, glycopeptides, fluoroquinolones, and aminoglycosides have been identified as major causes of nosocomial infections. Isolation of several MDR enterococci strains have a great concern due to the limitations in clinical use of antimicrobials, especially the use of their synergistic combinations which are often needed for the treatment of enterococcal infections [24].

There are some reports about increased resistance of enterococci to ciprofloxacin [25]. High resistance was observed to ciprofloxacin and nitrofurantoin in the present study. It may be due to the widespread use of these antibiotics for UTI as the first line treatment. Besides, the prevalence of vancomycin resistance in E. faecium (65\%) isolates was higher than in E. faecalis (26\%) isolates, which was in accordance with teicoplanin resistance pattern in these isolates. Moreover, both species of E. faecium and E. faecalis showed resistance to linezolid. Most of the E. faecium isolates were resistant to quinupristin- dalfopristin (83\%) and linezolid (60\%).

Besides, antibiotic-resistant Enterococcus spp. consisted potential virulence factors. It is worth noting that several of these enterococcal virulence traits are genetically transmissible through some genes located on specific regions of the genome, specifically in an area called the "pathogenicity islands" [17]. For example, exotoxin cytolysin (cyl) exhibits bacteriocidal characteristics towards Gramnegative bacteria and toxic properties towards erythrocytes, leukocytes, and macrophages. Aggregation substance (asa1) as an enterococcal surface protein participates in the formation of biofilm, which plays a significant role in the genetic material exchange between cells and improves their antibiotics resistance. In E. faecium strains, the presence of the esp gene is correlated with resistance to ampicillin, ciprofloxacin, and imipenem. Hyaluronidase (hyl) plays an important role in destroying mucopolysaccharides in cartilage and connective tissues, and subsequently, in spreading bacteria [26] Collagen-binding adhesin and gelatinase (gelE) also play an important role in increasing pathogenicity [27] However, acm (23, $64 \%)$ and hyl $(6,86 \%)$ were the most prevalent genes in E. faecalis, while the most prevalent virulence genes in E. faecium were cyt (7, $100 \%)$, gel $(30,97 \%)$ and asa $(29,97 \%)$. Only two (6\%) E. faecalis isolates carried asa and gel genes, and cyt was not detected in $E$. faecalis isolates. Other studies have also reported that the cyl and gel genes were more often carried by E. faecalis strains. But in the present study, cyt, asa, and gel were more common in E. faecium. 
The presence of multiple microorganisms at the site of infection usually causes biofilm-associated infections [28].

PFGE profiles indicated that high heterogeneity amongst $E$. faecalis and E. faecium clinical strains isolated from different patients in different wards, might be an indication of the possibility of crosstransmission of strains between patients in different wards within the hospitals. Similar studies findings exhibited a high genetic diversity amongst these isolates. Because of a high degree of differentiation between isolates, PFGE is considered as the "gold standard" for assessing hospital outbreaks[29]. Two common types, CT1 and CT2 contained some isolates from all three hospitals, suggesting the rotation of this bacterium in these three hospitals. The isolates typing results in this study showed that there was the possibility of inter-hospital transfer of strains.

\section{Conclusion}

In conclusion, in addition to identification of these strains, preventing and controlling the spread of multidrug-resistant enterococcal infections in hospitals require a coordinated effort between various departments, and this could only be achieved by monitoring the use of antibiotics, educating hospital staff, early detection and reporting by laboratories, and strict implementation of appropriate infection control measures.

\section{Acknowledgements}

The authors would like to thank the team in the microbiology departments of Iran University of Medical Science, Tehran, Iran.

\section{Conflict of Interest}

The authors have declared that no competing interests exist.

\section{Bibliography}

1. Kumpu Minna., et al. "Effect of live and inactivated Lactobacillus rhamnosus GG on experimentally induced rhinovirus colds: randomised, double blind, placebo-controlled pilot trial". Beneficial Microbes 6.5 (2015): 631-639.

2. Mascini EM and M J M Bonten. "Vancomycin-resistant enterococci: consequences for therapy and infection control". Clinical Microbiology and Infection 11 (2005): 43-56.

3. Schouten M A., et al. "Prevalence of vancomycin-resistant enterococci in Europe". European Journal of Clinical Microbiology and Infectious Diseases 19.11 (2000): 816-822.
4. Biedenbach Douglas J., et al. "Occurrence and antimicrobial resistance pattern comparisons among bloodstream infection isolates from the SENTRY Antimicrobial Surveillance Program (1997-2002)". Diagnostic Microbiology and Infectious Disease 50.1 (2004): 59-69.

5. Nelson R R S., et al. "Isolation and characterization of glycopeptide-resistant enterococci from hospitalized patients over a 30-month period". Journal of Clinical Microbiology 38.6 (2000): 2112-2116.

6. Feizabadi Mohammad Mehdi., et al. "Drug resistant patterns of enterococci recovered from patients in Tehran during 2000-2003". International Journal of Antimicrobial Agents 24.5 (2004): 521-522.

7. Christidou A., et al. "Emergence of vancomycin-resistant enterococci in a tertiary hospital in Crete, Greece: a cluster of cases and prevalence study on intestinal colonisation". Clinical Microbiology and Infection 10.11 (2004): 999-1005.

8. Chavers L S., et al. "Vancomycin-resistant enterococci: 15 years and counting". Journal of Hospital Infection 53.3 (2003): 159-171.

9. Klare I., et al. "Spread of ampicillin/vancomycin-resistant Enterococcus faecium of the epidemic-virulent clonal complex-17 carrying the genes esp and hyl in German hospitals". European Journal of Clinical Microbiology and Infectious Diseases 24.12 (2005): 815-825.

10. Fridkin Scott K., et al. "The effect of vancomycin and third-generation cephalosporins on prevalence of vancomycin-resistant enterococci in 126 US adult intensive care units". Annals of internal medicine 135.3 (2001): 175-183.

11. Talebi Malihe., et al. "Enterococcus faecium from meat, chicken and cheese". Jundishapur Journal of Microbiology 8.4 (2015).

12. Humphries Romney M., et al. "CLSI methods development and standardization working group best practices for evaluation of antimicrobial susceptibility tests". Journal of Clinical Microbiology 56.4 (2018).

13. Diekema Daniel J., et al. "Antimicrobial resistance trends and outbreak frequency in United States hospitals". Clinical Infectious Diseases 38.1 (2004): 78-85. 
14. Honarm Hamidreza., et al. "Evaluation of a PCR assay to detect enterococcus faecalis in blood and determine glycopeptides resistance genes: van A and van B". Iranian Journal of Medical Sciences 37.3 (2012): 194.

15. Shahbazi Shahla., et al. "Distribution of extended-spectrum $\beta$-lactam, quinolone and carbapenem resistance genes, and genetic diversity among uropathogenic Escherichia coli isolates in Tehran, Iran". Journal of Global Antimicrobial Resistance 14 (2018): 118-125.

16. Noohi Nasrin and Malihe Talebi. "Diversity and Genetic Clonality of Vancomycin and Gentamicin Resistant Enterococci Isolated from Healthy Humans in Iran". Journal of Medical Bacteriology 8.1-2 (2019): 27-36.

17. Abat Cédric., et al. "Low level of resistance in enterococci isolated in four hospitals, Marseille, France". Microbial Drug Resistance 22.3 (2016): 218-222.

18. Suppli Morten., et al. "Mortality in enterococcal bloodstream infections increases with inappropriate antimicrobial therapy". Clinical Microbiology and Infection 17.7 (2011): 10781083.

19. Seyfi Mahnaz., et al. "Anti-microbial resistance of enterococci isolated from urinary tract infections in Iran". (2008): 185-190.

20. ShanMuKhappa., et al. "Isolation, Identi Cation and Speciation of Enterococci by Conventional Method and Their Antibiogram". National Journal of Laboratory Medicine 4.2 (2015): 1-6.

21. Mutnick Alan H., et al. "Geographic variations and trends in antimicrobial resistance among Enterococcus faecalis and Enterococcus faecium in the SENTRY Antimicrobial Surveillance Program (1997-2000)". Diagnostic Microbiology and Infectious Disease 46.1 (2003): 63-68.

22. Duez Colette., et al. "The penicillin resistance of Enterococcus faecalis JH2-2r results from an overproduction of the low-affinity penicillin-binding protein PBP4 and does not involve a psrlike geneThe GenBank accession numbers for the sequences reported in this paper are Y17797 for the 8. $4 \mathrm{~kb}$ segment of pDML521; AJ290435 for pbp4 of E. faecalis JH2-2; AJ276231 and AJ276232 for the psr-like gene of E. faecalis JH2-2 or JH22r, respectively". Microbiology 147.9 (2001): 2561-2569.
23. Jadhav R K., et al. "Bacterial urinary tract infections in canines and their management". North-East Veterinarian 11.2 (2011): 29-16.

24. Maschieto Andresa., et al. "Antimicrobial resistance of Enterococcus sp. isolated from the intestinal tract of patients from a university hospital in Brazil”. Memórias do Instituto Oswaldo Cruz 99.7 (2004): 763-767.

25. Kaçmaz Birgül and Altan Aksoy. "Antimicrobial resistance of enterococci in Turkey". International journal of Antimicrobial Agents 25.6 (2005): 535-538.

26. Chajęcka-Wierzchowska., et al. "Virulence factors of Enterococcus spp. presented in food". LWT 75 (2017): 670-676.

27. M'hir Sana., et al. "Technological, functional and safety aspects of enterococci in fermented vegetable products: a mini-review". Annals of Microbiology 62.2 (2012): 469-481.

28. Golob Majda., et al. "Antimicrobial resistance and virulence genes in Enterococcus faecium and Enterococcus faecalis from humans and retail red meat". BioMed research international 2019 (2019).

29. Weng Poh Leng., et al. "High genetic diversity of Enterococcus faecium and Enterococcus faecalis clinical isolates by pulsedfield gel electrophoresis and multilocus sequence typing from a hospital in Malaysia". BioMed research international 2013 (2013).

\section{Volume 4 Issue 7 July 2021}

(C) All rights are reserved by Arezoo Asadi., et al. 